\title{
Costs of health care across primary care models in Ontario
}

Maude Laberge ${ }^{1,2^{*}}$ (D) Walter P Wodchis ${ }^{3,4,5}$, Jan Barnsley ${ }^{3,4}$ and Audrey Laporte 2,3

\begin{abstract}
Background: The purpose of this study is to analyze the relationship between newly introduced primary care models in Ontario, Canada, and patients' primary care and total health care costs. A specific focus is on the payment mechanisms for primary care physicians, i.e. fee-for-service (FFS), enhanced-FFS, and blended capitation, and whether providers practiced as part of a multidisciplinary team.

Methods: Utilization data for a one year period was measured using administrative databases for a $10 \%$ sample selected at random from the Ontario adult population. Primary care and total health care costs were calculated at the individual level and included costs from physician services, hospital visits and admissions, long term care, drugs, home care, lab tests, and visits to non-medical health care providers. Generalized linear model regressions were conducted to assess the differences in costs between primary care models.

Results: Patients not enrolled with a primary care physicians were younger, more likely to be males and of lower socio-economic status. Patients in blended capitation models were healthier and wealthier than FFS and enhanced-FFS patients. Primary care and total health care costs were significantly different across Ontario primary care models. Using the traditional FFS as the reference, we found that patients in the enhanced-FFS models had the lowest total health care costs, and also the lowest primary care costs. Patients in the blended capitation models had higher primary care costs but lower total health care costs. Patients that were in multidisciplinary teams (FHT), where physicians are also paid on a blended capitation basis, had higher total health care costs than non-FHT patients but still lower than the FFS reference group. Primary care and total health care costs increased with patients' age, morbidity, and lower income quintile across all primary care payment types.
\end{abstract}

Conclusions: The new primary care models were associated with lower total health care costs for patients compared to the traditional FFS model, despite higher primary care costs in some models.

Keywords: Health care costs, Primary care, Payment systems: FFS/capitation/DRGs/Risk Adjusted Payments etc.

\section{Background}

Physician payments and methods of remuneration have been topics of increasing interest as policy makers search for the "right" payment policy to balance physicians', patients', and payers' interests [1]. Physicians may be incentivized to provide fewer or more services depending on the payment methods, yet how sensitive they are to the financial incentives may depend on their level of altruism [2].

\footnotetext{
* Correspondence: Maude.laberge@fsa.ulaval.ca

${ }^{1}$ Department of Operations and Decision Systems, Faculty of Administrative

Sciences, Université Laval, 2325 rue de la Terrasse, \#2519, Quebec City

G1V0A6, Quebec, Canada

${ }^{2}$ Canadian Centre for Health Economics, Toronto, Canada

Full list of author information is available at the end of the article
}

In most countries, payers also have a responsibility towards maintaining and improving the health of the population within budget constraints. In this context, payers - in many cases, governments - introduced different ways of remunerating physicians, particularly in primary care. One example is the Quality and Outcomes Framework (QOF) in the UK, a program that blends capitation payment with incentives and rewards for primary care physicians to meet performance targets. Most of these targets are related to the management of common chronic conditions, and the delivery of preventive services [3]. The implementation of a new remuneration scheme for primary care physicians such as the QOF is 
typically associated with an increase in the spending in primary care.

The evidence suggests that the processes of strengthening primary care with measures including higher investments and chronic disease management programs would be more than compensated for by avoidance of a population's deterioration in health and of complications that result in higher utilization of expensive health care services [4-8].

In Canada, where the responsibility for health care is decentralized to the provinces, the government of Ontario (the most populous province) initiated a reform of primary care in the early 2000s. The reform consisted of the gradual introduction of models characterized by mixed payment mechanisms as well as practice requirements and an enrollment process, formally defining the physician-patient relationship [9]. The reform had multiple aims including improving access to and quality of primary care and controlling health care costs. Primary care services include diagnostic and treatment for the majority of health conditions, patient education, health promotion and disease prevention services. These services are mainly delivered by family physicians, nurses, and pharmacists. In Canada, family physicians are the entry point into the health care system and act as gatekeepers to access to specialized services; primary care services are meant to provide the majority of health care needs to the population. The context at the time was one of primary care physician shortage, where Ontarians reported experiencing difficulties finding a primary care physician, and when they had one, being able to see their physician in a timely matter $[10,11]$. The vast majority of primary care physicians were remunerated on a feefor-service (FFS) basis. The FFS method is considered to have important drawbacks not only because it provides an incentive to over-supply care, but also, because remuneration (at least in Ontario) is disconnected from the quality of care provided and may inhibit collaboration amongst health care providers [12, 13]. On the other hand, FFS physicians provide more services than physicians paid by capitation or salary [14], which should be associated with better access.

Between 2001 and 2007, the Ontario government introduced new primary care models offering physicians the option of shifting away from FFS towards enhancedFFS and blended capitation payment mechanisms. Both of these payment mechanisms consisted of mixing FFS payment and capitation payment with bonus and incentive payments for the delivery of preventive services (such as cancer screening) and chronic disease management (such as for diabetes).

There are currently two enhanced-FFS models: the Comprehensive Care Model (CCM) and the Family Health Group (FHG); the latter requires physicians to practice in a group of at least three physicians. Because of the differences in payment and practice structures, CCM and FHG are considered separately. There are also two blended capitation payment models: the Family Health Network (FHN) and the Family Health Organization (FHO). FHOs have a higher capitation rate than FHNs, which reflects a larger basket of primary care services included in the rate. Services provided outside of the basket are compensated via FFS; FHN and FHO physicians also receive $15 \%$ of the fees for services that are included in the capitation rates. In addition, FHOs and FHNs can apply to the Ontario Ministry of Health and Long Term Care (MOHLTC) to form a Family Health Team (FHT). FHTs receive additional funding to hire other types of health care professionals and support an interdisciplinary team environment. There are no intended differences in physicians' payment whether the practice is a FHT or not. Because the payments are different between $\mathrm{FHO}$ and $\mathrm{FHN}$, they are considered separate primary care models. Each of the FHO and FHN models are also distinguished by whether they practice as a FHT.

In the Canadian context where health care services are provided free at the point of services, it is assumed that the utilization of health care services is determined by one's needs, as opposed to ability to pay. In general, older individuals and individuals in poorer health have a higher utilization of health care services. Others have shown that a lower socio-economic status (SES) is associated with a higher utilization, even after adjusting for health status [15].

This study examines the associations between the primary care model a patient belongs to, and patients' primary care costs and total health care costs, using the traditional FFS model as the reference.

\section{Methods}

All Ontario residents (13.6 million people in 2013) are covered by the Ontario Health Insurance Plan (OHIP) for medically necessary services. Our cohort population for this study consisted of a 10\% random sample of the adult population who had a valid health care card at the index date (April 1, 2012). Individuals were excluded from the study sample if they incurred zero primary care costs (meaning that they did not use physician services) or if they died during the study period (April 12012 to March 21st 2013).

\section{Data source and variables}

Encrypted data for this cross-sectional study were obtained from administrative databases at the Institute for Clinical Evaluative Sciences (ICES). The study received ethics approval from the Research Ethics Boards of the University of Toronto and the Sunnybrook Health 
Sciences Centre. The sample was extracted from the adult population in the Ontario Registered Persons Database (RPDB) which contains basic demographic information on individuals. Using unique patient identifiers, the ICES Key Numbers (IKN), patients' enrolment data at the beginning of the study period from the Client Agency Program Enrolment (CAPE) database were used to link patients to their primary care physicians and to the corresponding payment model to which the physician belonged. Patients who were not formally enrolled with a physician were considered as a separate group the not enrolled. The IKN were also used to retrieve each individual's health services utilization. All health care services paid for by the MOHLTC were included: physician services were extracted from the OHIP database; general hospital services from the Canadian Institute for Health Information Discharge Abstract Database, the Same Day Surgery Database, and the National Ambulatory Care Reporting System; drugs for adults on social assistance and for people aged 65 and over from the Ontario Drug Benefit Plan database; home care services from the Home Care database; lab and non-physician billings from the OHIP. Utilization from long term episodes of care were combined to include residents of nursing homes and of specialized hospitals with data from the Complex Continuing Care database, the Ontario Mental Health Reporting System database and the Long Term Care database.

The two outcome variables were primary care costs (PCC) and total health care costs (THCC).

Costs were calculated for each individual in the study population based on the individual's utilization of health care services during the study period and the prices of services, as paid by the MOHLTC. Algorithms for the prices of health care services have been previously developed by a team of Ontario researchers and implemented at ICES [16]. Primary care costs were composed of the prices and quantity of each service billed by primary care physicians to OHIP as well as capitation costs, and shadow billing costs. The capitation rates depended on the primary care model that the physician belonged to.

Total health care costs were calculated based on the utilization of health care services that each individual made. They included services paid for on a fee schedule as well as institutional care. Costs for institutional care were adjusted for the resource intensity weight of the care setting. In the case of long term care, costs were based on a per diem fee paid by the MOHLTC.

The costs associated with the payments for the establishment and operations of FHTs, as well as performance payments to primary care physicians were not available for inclusion at the individual patient level.
The independent variables of interest were the primary care models that physicians can belong to: CCM, FHG, FHN, FHO, FHT-FHN, FHT-FHO, using the FFS as the reference group.

Since patients may be differentially distributed across models [17], the statistical models controlled for patient characteristics that could affect health care utilization and costs. Explanatory variables included patient's age (continuous variable), sex (dichotomous variable), and socio-economic status, using the income quintile as a proxy (based on postal codes with the lowest quintile (1) as the reference group), the Adjusted Clinical Group (ACG ${ }^{\circ}$ ) weight, and the Rurality Index of Ontario (RIO) score for the practice location of the primary care physician.

The $\mathrm{ACG}^{\circ}$ system is a measure of case-mix developed by the Johns Hopkins University to reflect patients' health care needs based on the combination of their diagnostics, age and sex [18]. The $\mathrm{ACG}^{\bullet}$ weight ranges from 0.000 to 4.666 , with a higher weight representing a poorer health status. There are only a few scores over 1.000 , and these reflect a high level of complexity with a combination of: at least 2 major adjusted diagnostic groups (ADGs); at least 6 other ADG combinations; and an age over 34. The algorithm accounts for the mix of diagnosis over a defined period of time and across health care settings. The $\mathrm{ACG}^{\circ}$ has been tested and it was validated as a predictor of utilization and mortality in Canada [19-21]. A recent review of various morbidity measures suggests that the $\mathrm{ACG}^{\circ}$ system is the strongest in predicting health care utilization [22]. The RIO is a continuous variable that takes a value between 0 and 100 , with lower values indicating an urban location and it adjusts for the geographic location of the primary care practice. The RIO is a measure that was developed by the Ontario Medical Association for Ontario communities [23]. The RIO includes the following 10 variables: travel time to nearest basic referral center, travel time to nearest advanced referral center, community population, number of active general practitioners (GP), population-to-GP ratio, presence of a hospital, availability of ambulance services, social indicators, weather conditions, and selected services.

\section{Statistical Analysis}

There are multiple approaches to the analysis of cost data, with the most common being the OLS with a log transformation and the generalized linear model [GLM] [24-28]. Although the OLS with log transformation is widely known and used, it does not eliminate heteroscedasticity and the retransformation could lead to bias. The GLM is a preferred approach in the presence of heteroscedasticity in a statistical model with multiple covariates. The GLM was selected for this study after the 
Breusch-Pagan test found heteroscedasticity and because of other advantages of the GLM. The GLM takes into account heteroscedasticity, and does not require retransformation so as to express estimates in dollar amounts and accommodates skewness, which is typical of health care cost data [29-31]. This method does require specifying a distribution for the mean-variance relationship and a link function. Gaussian, Poisson, Gamma and inverse Gaussian distributions for the mean-variance relationship were tested with the modified Park test $[24,30]$. The results reported here are based on the GLM with an identity link and a Gaussian family based on the results from the Park test.

Two regressions were conducted: the first regression modelled patient primary care costs, and dummy variables for the models; the second analysis examined total health care costs as a function of the primary care model of the primary care physician and also of the other explanatory variables identified.

Regression models for both primary care and total health care costs were defined as:

$$
\begin{aligned}
& \operatorname{Cost}_{\mathrm{i}}=\beta_{0}+\beta_{1} \mathrm{CCM}_{\mathrm{i}}+\beta_{2} \mathrm{FHG}_{\mathrm{i}}+\beta_{3} \mathrm{FHN}_{\mathrm{i}}+\beta_{4} \mathrm{FHO}_{\mathrm{i}} \\
& +\beta_{5} \text { FHT-FHN } i+\beta_{6} \text { FHT-FHO }+\beta_{7} \text { RIO }_{i} \\
& +\beta_{8} \text { ACGweight }_{\mathrm{i}}+\beta_{9} \text { age }_{\mathrm{i}}+\beta_{10} \text { sex }_{\mathrm{i}} \\
& +\beta_{7} \text { income }_{\text {quintile }}+\varepsilon_{\mathrm{i}}
\end{aligned}
$$

Where: Cost $_{i}$ is either the primary care or the total health care cost of the services for patient $i$ for a 12 month period; $\beta_{0}$ is the intercept; $\mathrm{CCM}_{\mathrm{i}}, \mathrm{FHG}_{\mathrm{i}}, \mathrm{FHN}_{\mathrm{i}}$, $\mathrm{FHO}_{\mathrm{i}}$, FHT-FHN $\mathrm{i}_{\mathrm{i}}$, and FHT-FHO $\mathrm{F}_{\mathrm{i}}$ are dichotomous variables for the primary care models, using FFS as the reference group; $\mathrm{RIO}_{i}$ is the value of the RIO of the practice the patient $\mathrm{i}$ belongs to; $\mathrm{age}_{\mathrm{i}}$, $\operatorname{sex}_{\mathrm{i}}$, and income_quintile $_{i}$ are the adjustments for the patient's age, sex, and neighborhood income quintile; $\varepsilon_{\mathrm{i}}$ is the error term for patient $\mathrm{i}$.

\section{Results}

The sample contained 1,133,645 observations. Table 1 describes the characteristics of the patients in the sample as a whole and according to primary care model, including the average costs, broken down by health care sector and types of services.

The highest health care costs are hospital costs, followed by physician costs. The results in Table 1 show that there are significant differences in the characteristics of patients across models compared to FFS patients and significant differences in the costs as well.

The sample size was reduced to 1,094,687 because of missing data on the RIO variable for patients across all models. The marginal effects in dollars of the explanatory variables were computed from the GLM models, using the method described by Jones et al. (2013) and are reported in Table 2. The marginal effects assessed at the mean of the explanatory variables are reported below.

The effect of the primary care model on a patient's costs is measured through the variables CCM, FHG, FHN, FHO, FHT-FHN, and FHT-FHO. Compared to FFS patients, primary care costs for one year are on average $\$ 32$ lower for CCM patients and \$13 lower for FHG patients. They are $\$ 16$ higher for FHO patients. The primary care costs of patients who are not enrolled but who are seeing a physician who is in a patient enrolment model are not significantly different from those of patients seeing FFS physicians. Compared to FFS patients, total health care costs are lower on average by $\$ 658, \$ 667, \$ 446, \$ 485, \$ 433$, and $\$ 392$ for CCM, FHG, FHN, FHO, FHT-FHN, and FHT-FHO patients respectively. They are $\$ 130$ higher for not-enrolled patients.

Although the $\mathrm{ACG}^{\circ}$ weight, age and sex control for a patient's health status, the SES of a patient still has a significant effect on both primary care costs and total health care costs, which are incrementally lower with higher SES, as measured with the neighborhood income quintile.

\section{Discussion}

The results suggest there are statistically significant differences in both total health care and primary care costs across primary care models after controlling for patient health status and SES as well as practice location. The discussion is structured as follows: first, a discussion of the primary care costs; then, a discussion of the total health care costs; finally a review of some limitations of the study that may explain some of the results obtained.

\section{Primary care costs}

The lower primary care costs of enhanced-FFS patients (\$32 lower for CCM patients and \$13 lower for FHG patients) appear counter-intuitive to the fact that the payment mechanisms are relatively similar, in that CCM and FHG are based on FFS payment. It may be that the small incentives for enrolment of patients in CCM and FHG, as well as the incentives for preventive services and chronic disease management are effective at supporting higher quality of care, continuity of the patientphysician relationship and that these indirectly support lower primary care needs and visits compared to the FFS patients [32]. Non-enrolled patients had primary care costs that were not significantly different from those of FFS patients. This was an expected result in that payments to physicians for these patients are the same as for patients of FFS physicians. Because these patients were not enrolled to the physician that they were seeing, they were maybe also less likely to have a continuous 


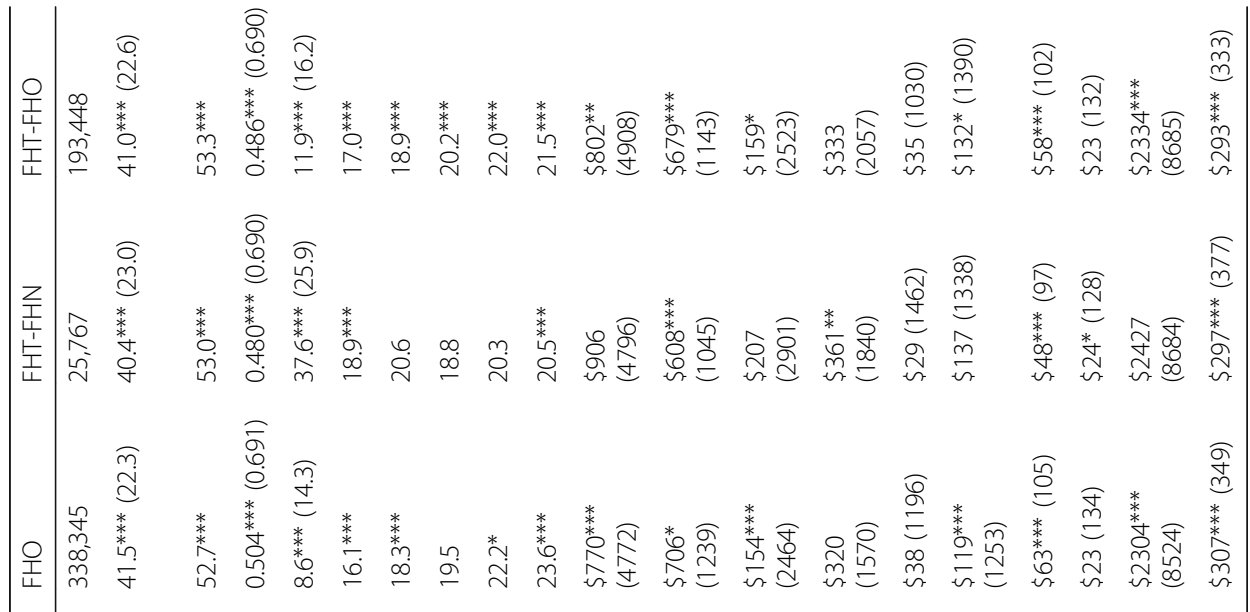

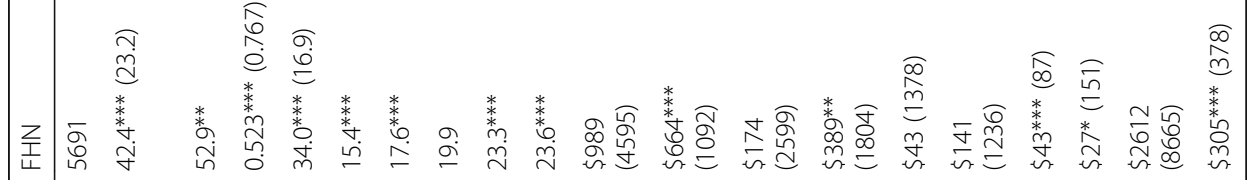

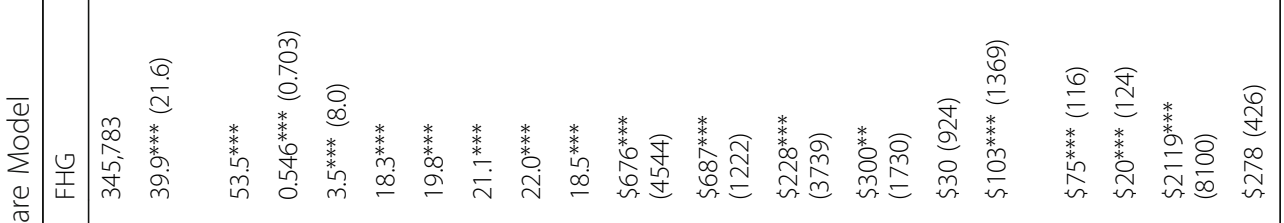

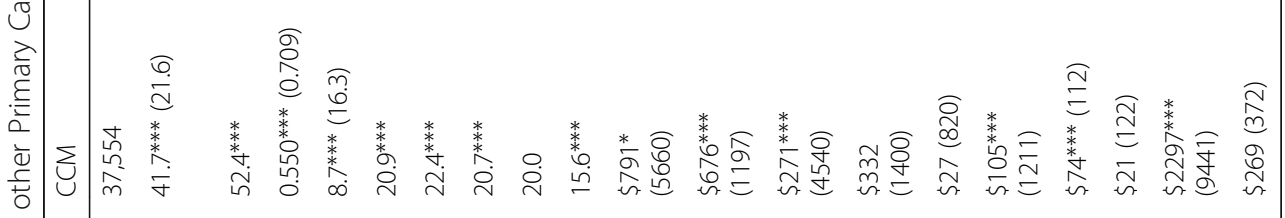

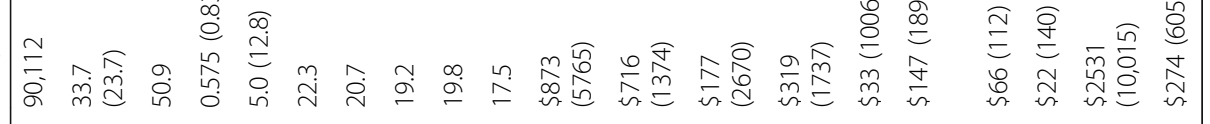


Table 2 The Average Marginal Effect of Primary Care Models on One-Year Primary Care Costs and on Total Health Care Costs

\begin{tabular}{|c|c|c|}
\hline Variable & $\begin{array}{l}\text { Primary Care } \\
\text { Cost (in \$) }\end{array}$ & $\begin{array}{l}\text { Total Health Care } \\
\text { Cost (in \$) }\end{array}$ \\
\hline \multicolumn{3}{|l|}{$N=1,094,687$} \\
\hline \multicolumn{3}{|l|}{ Practice Characteristics: } \\
\hline FFS & reference & reference \\
\hline Not enrolled & -5 & $130^{*}$ \\
\hline Enhanced FFS - CCM & $-32^{* * *}$ & $-658^{* * *}$ \\
\hline Enhanced group FFS - FHG & $-13^{* *}$ & $-667^{* * *}$ \\
\hline $\mathrm{FHN}$ & 0.1 & $-446^{* * *}$ \\
\hline $\mathrm{FHO}$ & $16^{* * *}$ & $-485^{* *}$ \\
\hline $\mathrm{FHT}-\mathrm{FHN}$ & 2 & $-433^{* * *}$ \\
\hline $\mathrm{FHT}-\mathrm{FHO}$ & 5 & $-392^{* * *}$ \\
\hline Practice RIO & $0.3^{* * *}$ & $6.8^{* * *}$ \\
\hline \multicolumn{3}{|l|}{ Patient Characteristics: } \\
\hline Patient age & $4^{* * *}$ & $61^{* * *}$ \\
\hline Patient female & $48^{* * *}$ & $-101^{* * *}$ \\
\hline ACG ${ }^{\circledast}$ weight & $128^{* * *}$ & $2947^{* * *}$ \\
\hline Income quintile 1 & Reference & Reference \\
\hline Income quintile 2 & $-17^{* * *}$ & $-325^{* * *}$ \\
\hline Income quintile 3 & $-21^{* * *}$ & $-436^{* * *}$ \\
\hline Income quintile 4 & $-27^{* * *}$ & $-517^{* * *}$ \\
\hline Income quintile 5 & $-33^{* * *}$ & $-607^{* * *}$ \\
\hline
\end{tabular}

***indicates significance at $p<0.001 ; * *$ indicates significance at $p<0.01$; *indicates significance at $p<0.05$

relationship with the physician and a monitoring and management of their health.

FHO patients had primary care costs that were $\$ 16$ higher than FFS patients. These higher primary care costs for patients in a blended-capitation models could appear counterintuitive in relation to the theoretical literature, which supports capitation remuneration as a form of cost containment [33]. But this significant difference in primary care cost can be explained by a policy objective associated with the FHO model of moving physicians away from the FFS payment model. To attract physicians into a prospective and risk-sharing payment mechanism, the remuneration had to be higher than the expected remuneration on a FFS basis, all else being equal. The higher adjusted per-patient costs reflect that the FHO payments are in fact higher than what would have been paid to primary care physicians in the FFS model. The FHN was a blended capitation model introduced before the FHO and it was seen as unsuccessful; few physicians formed FHNs because the capitation rates were considered too low [34]. The results in Table 2 in regards to FHN patients are consistent with the idea that the FHN capitation rates were not high enough to entice physicians to join or form FHNs: primary care costs of
FHN patients are not significantly different from those of FFS patients.

Finally, the primary care costs of FHT patients, both FHT-FHNs and FHT-FHOs were not significantly different from those of FFS patients. These results may reflect the fact that the additional funding provided by the MOHLTC for the establishment and operations of FHTs were not available for inclusion. The funding supports the hiring of various health care providers, which may substitute for some of the care that would be delivered by physicians in non-FHT practices. Hence the primary costs of FHT patients were under-estimated in our analysis and the observed higher costs, could be significant if the additional funding were included. In 2014, the average operational budget of FHTs was $\$ 1.75$ million with a range from $\$ 0.27$ million to $\$ 21.7$ million, and these budgets were considered as having been stable in the past few years. The total budget from the MOHLTC to FHTs was \$344 million [personal communication, Association of Family Health Teams of Ontario, January 18th, 2016 \& Ministry of Health and Long Term Care, Primary Health Care Branch, January 21st, 2016]. The MOHLTC also reports that over 3 million patients are enrolled in FHTs. Based on these numbers from the total budget and the number of patients enrolled, a rough estimate of the additional FHT per patient cost would be $\$ 114.67$. Hence, it could be extrapolated that the primary care costs of FHT patients are likely to be significantly higher than those of FFS patients.

Primary care costs increased with higher rurality of the primary care practice, with a patient's age, and higher morbidity reflecting higher needs in older and sicker patients. Being female was associated with on average $\$ 48$ higher primary care costs also reflecting the higher medical needs of women. Results also showed an income gradient in that primary care decrease with higher income neighborhood quintile. Higher utilization of primary care services in lower income population, even after adjusting for morbidity has been observed elsewhere in Canada [15]; it may partly be attributable to the lower health literacy and capacity to benefit from a physician visit in patients of lower SES [35].

\section{Total health care costs}

Total health care costs were significantly lower in patients of all primary care models compared to FFS patients. The not enrolled patients had higher total health care costs.

Compared to FFS, the fact that total health care costs were lower in blended capitation model patients may appear surprising: the theoretical literature suggests that a capitation payment to primary care physicians provides an incentive for an under-provision of care and a shift towards other levels of care that are more expensive, 
such as specialists' care or hospital care [33]. In fact, Allard et al. (2011) suggest that FFS would be the optimal payment system if primary care physicians all had a high diagnostic ability and were relatively altruistic in their desire to provide high-quality care, or if patients' outcomes were measured and considered in the payments. However, it is more likely that there is heterogeneity amongst physicians in relation to those two aspects. In addition, in a context such as the one in Ontario, where primary care physicians have various payment options, physicians may choose the primary care model that is most beneficial in relation to their skills and altruism levels, given the characteristics of their patient population (which they can also choose). In fact, other researchers who examined physicians' levels of altruism found heterogeneity and differences in optimal payment methods, depending on a physician's altruism [36-39]. It has also been found that quantities of care provided were closer to the optimum for subjects with higher levels of altruism and that mixed payment alleviated the negative effects associated with the incentives in each type of payment mechanism [2].

Although the purpose of this study was to analyze the costs using the traditional FFS as the reference group, examining the results between the models reveals some interesting findings. Within models, the differences in total health care costs were higher in enhanced-FFS models, i.e. the CCM (\$-658) and the FHG (\$-667) patients whereas the total health care costs of patients in blended capitation models were lower than those of FFS patients but not by as much: $\$-446$ for FHN patients, \$-485 for FHO patients, \$-433 for FHN-FHT patients, and \$-392 for FHO-FHT patients. These results are consistent with those of a study where patients of enhanced-FFS physicians had the lowest potentially preventable hospital utilization, followed by patients of physicians in blended capitation models [40]. The fact that total health care costs are the lowest in the enhancedFFS models is consistent with the idea that physicians' levels of altruism and ability may not be randomly distributed across primary care models.

Including the estimated $\$ 114.67$ additional cost per FHT patient to the patients' total health care costs would reduce the differences between FFS patients and FHT patients (FHT-FHO and FHT-FHN). However, FHT patients are likely to still have significantly lower total health care costs than FFS patients. The FHTs were designed to offer more comprehensive services with a range of services to more complex patients. However, our results, adjusting for patient characteristics suggest that although FHTs appears to be cost saving compared to FFS patients, FHT patients are not less costly than patients in other primary care models. It may be that there is a misalignment between the characteristics of the patients treated in the
FHTs and the services offered. The results in Table 1 do show that the average $\mathrm{ACG}^{\bullet}$ weight of FHT patients is significantly lower compared to the average of FFS patients and it is also lower than the averages of patients in all other models. The income quintile distribution shows that FHT patients are also wealthier.

In terms of control variables, the effects observed in primary care costs are accentuated in total health care costs except for the sex variable. As expected, total health care costs increased with practice rurality, with the patient's age and $\mathrm{ACG}^{\circ}$ weight, and decreased with higher SES. Surprisingly, being female was associated with lower total health care costs. It may be that women use more preventive primary care and, when controlling for morbidity with the $\mathrm{ACG}^{\circ}$, are better able to manage their health conditions than males.

The large effect of SES (on average, a \$607 lower total health care cost for a patient from the highest neighborhood income quintile compared to the total health care cost of a patient from the lowest neighborhood income quintile) may reflect that people of lower SES have a lower health literacy and capacity to manage their health. It may also be that the $\mathrm{ACG}^{\circ}$ does not accurately capture the severity of conditions and the severity of diseases afflicting people in lower SES is higher.

\section{Limitations}

The limitations of this study include those related to outcomes examined and the availability of the data. Specifically, because we could only obtain limited data on the additional funding provided to FHTs, we could only provide estimates as an average for the patient level. Drug costs only included those paid for by the MOHLTC for: people 65 and older, residents of longterm care facilities or a home for special care, and people receiving social assistance, and people registered through the Trillium Drug Program (eligible only if their drug costs are considered high relative to their income). Primary care funding related to performance targets were not included in patient level costs and but these would affect costs across the primary care models to a similar extent, with the exception of the FFS patients, for which physicians do not receive any bonus payments.

Even though total health care costs at the patient level were found to be lower in the new primary care models compared to the traditional FFS model, these would need to be further examined in relation to the health outcomes of the patients.

\section{Conclusion}

Blended capitation models were associated with higher primary care costs. However, our results suggest that 
these were more than compensated for with lower total health care costs. More research is needed to better understand the causes for higher total health care costs associated with patients for whom physicians are paid on a FFS basis and to understand potential implications in terms of quality of care and patient outcomes.

\section{Abbreviations}

ACG®: Adjusted Clinical Group; CAPE Client: Agency Program Enrolment; CCM: Comprehensive Care model; FFS: Fee-For-Service; FHG: Family Health Group; FHN: Family Health Network; FHO: Family Health Organization; FHT: Family Health Team; GLM: Generalized Linear Model; ICES: Institute for Clinical Evaluative Sciences; IKN: ICES Key Number; MOHLTC: Ministry of Health and Long Term Care; OHIP: Ontario Health Insurance Plan; PCC: Primary Care Costs; QOF: Quality and Outcomes Framework; RIO: Rurality Index of Ontario; RPDB: Registered Persons Database; SES: SocioEconomic Status; THCC: Total Health Care Costs

\section{Acknowledgements}

The Institute for Clinical Evaluative Sciences (ICES) provided the dataset for the study.

\section{Availability of data and materials}

The data used for this study contains deidentified health information from multiple administrative databases in Ontario. These data are protected by the Health Information Protection Act and are not available.

\section{Authors' contributions}

$M L$ designed the study, conducted the statistical analysis and wrote the first draft of the manuscript. WPW provided guidance on the development of the study design, access to the data and critical review of the manuscript. JB and AL contributed to the study design and provided substantial revising of the manuscript. All authors have given approval to the final manuscript.

\section{Ethics approval and consent to participate}

The study received approval from the Research Ethics Board of the University of Toronto; protocol reference \#29686. Access to the data was provided by the Institute for Clinical Evaluative Sciences (ICES) and all analyses were conducted in the secure ICES environment.

\section{Competing interests}

The authors declare that they have no competing interests.

\section{Publisher's Note}

Springer Nature remains neutral with regard to jurisdictional claims in published maps and institutional affiliations.

\section{Author details}

${ }^{1}$ Department of Operations and Decision Systems, Faculty of Administrative Sciences, Université Laval, 2325 rue de la Terrasse, \#2519, Quebec City G1V0A6, Quebec, Canada. ${ }^{2}$ Canadian Centre for Health Economics, Toronto, Canada. ${ }^{3}$ Institute of Health Policy, Management and Evaluation, University of Toronto, Toronto, Canada. ${ }^{4}$ Institute for Clinical Evaluative Sciences, Toronto, Canada. ${ }^{5}$ Toronto Rehabilitation Institute, Toronto, Canada.

Received: 23 January 2017 Accepted: 17 July 2017

Published online: 01 August 2017

\section{References}

1. Bekelman JE, Epstein AJ, Emanuel EJ. Getting the Next Version of Payment Policy "Right" on the Road Toward Accountable Cancer Care. Int J Radiat Oncol Biol Phys. 89(5):954-7.

2. Brosig-Koch J, Hennig-Schmidt H, Kairies-Schwarz N, Wiesen D. The Effects of Introducing Mixed Payment Systems for Physicians: Experimental Evidence. Health Econ. Feb. 2017;26(2):243-62.

3. Campbell SM, Kontopantelis E, Hannon K, Burke M, Barber A, Lester HE. Framework and indicator testing protocol for developing and piloting quality indicators for the UK quality and outcomes framework. BMC Fam Pract. 2011;12:85.
4. Bodenheimer T, Wagner EH, Grumbach K. Improving primary care for patients with chronic illness. JAMA. Oct. 2002;288(14):1775-9.

5. Fries JF, et al. Reducing Health Care Costs by Reducing the Need and Demand for Medical Services. N Engl J Med. Jul. 1993;329(5):321-5.

6. Wagner EH, Sandhu N, Newton KM, McCulloch DK, Ramsey SD, Grothaus LC. Effect of improved glycemic control on health care costs and utilization. JAMA. Jan. 2001;285(2):182-9.

7. Phillips CD, Hawes C, Fries BE. Reducing the use of physical restraints in nursing homes: will it increase costs? Am J Public Health. Mar. 1993;83(3):342-8.

8. Lorig K, et al. Chronic disease self-management program: 2-year health status and health care utilization outcomes. Med Care. 2001:39(11);1217-23.

9. Hutchison B, Glazier R. Ontario's Primary Care Reforms Have Transformed The Local Care Landscape, But A Plan Is Needed For Ongoing Improvement. Health Aff. (Millwood). 2013;32(4):695-703.

10. Schoen C, Osborn R, Doty MM, Bishop M, Peugh J, Murukutla N. Toward Higher-Performance Health Systems: Adults. Health Care Experiences In Seven Countries, 2007', Health Aff. (Millwood). 2007;26(6):w717-34.

11. Schoen C, Doty MM. Inequities in access to medical care in five countries: findings from the 2001 Commonwealth Fund International Health Policy Survey. Health Policy. 67(3):309-22.

12. Berenson RA, Rich EC. US Approaches to Physician Payment: The Deconstruction of Primary Care. J Gen Intern Med. 2010;25(6):613-8.

13. Pullon S, McKinlay E, Dew K. Primary health care in New Zealand: the impact of organisational factors on teamwork. Br J Gen Pract. 2009;59(560):191-7.

14. Gosden T, et al. Capitation, salary, fee-for-service and mixed systems of payment: effects on the behaviour of primary care physicians. Cochrane Database Syst Rev. 2000;3

15. Roos LL, Walld R, Uhanova J, Bond R. Physician Visits, Hospitalizations, and Socioeconomic Status: Ambulatory Care Sensitive Conditions in a Canadian Setting. Health Serv Res. 2005;40(4):1167-85

16. Wodchis W, Bushmeneva K, Nikitovic M, McKillop I. Guidelines on PersonLevel Costing Using Administrative Databases in Ontario, vol. 1. Toronto: Health Systems Performance Research Network; 2013.

17. Rudoler D, Laporte A, Barnsley J, Glazier RH, Deber RB. Paying for primary care: A cross-sectional analysis of cost and morbidity distributions across primary care payment models in Ontario Canada. Soc Sci Med. 2015;124:18-28.

18. Starfield B, Weiner J, Mumford L, Steinwachs D. Ambulatory care groups: a categorization of diagnoses for research and management. Health Serv Res. 1991;26(1):53-74.

19. Glazier RH, Klein-Geltink J, Kopp A, Sibley LM. Capitation and enhanced fee-for-service models for primary care reform: a population-based evaluation. Can Med Assoc J. 2009;180(11):E72-81.

20. Reid RJ, Roos NP, MacWilliam L, Frohlich N, Black C. Assessing Population Health Care Need Using a Claims-based ACG Morbidity Measure: A Validation Analysis in the Province of Manitoba. Health Serv Res. 2002;37(5): 1345-64.

21. Sibley L, Glazier R, Hutchison B. Measuring the casemix of physician practices in primary-care reform models in Ontario, Canada. BMC Health Serv Res. 2011:11(Suppl 1):A22.

22. Huntley AL, Johnson R, Purdy S, Valderas JM, Salisbury C. Measures of Multimorbidity and Morbidity Burden for Use in Primary Care and Community Settings: A Systematic Review and Guide. Ann Fam Med. 2012; 10(2):134-41.

23. B. Kralj, 'Measuring Rurality - RIO2008_BASIC: Methodology and Results', Ontario Medical Association, 2009.

24. Buntin MB, Zaslavsky AM. Too much ado about two-part models and transformation?: Comparing methods of modeling Medicare expenditures. J Health Econ. 2004;23(3):525-42.

25. Garrido MM, Deb P, Burgess JF, Penrod JD. Choosing Models for Health Care Cost Analyses: Issues of Nonlinearity and Endogeneity. Health Serv Res. 2012;47(6):2377-97.

26. Moran JL, Solomon PJ, Peisach AR, Martin J. New models for old questions: generalized linear models for cost prediction. J Eval Clin Pract. 2007;13(3): $381-9$.

27. Vavken P, Pagenstert G, Grimm C, Dorotka R. 'Does increased health care spending afford better health care outcomes? Evidence from Austrian health care expenditure since the initiation of DRGs.', Swiss Medical Weekly. 2012;142:w13589.

28. Mihaylova B, Briggs A, O'Hagan A, Thompson SG. Review of Statistical Methods for Analysing Healthcare Resources and Costs. Health Econ. 2011; 20(8):897-916 
29. Blough DK, Ramsey SD. Using Generalized Linear Models to Assess Medical Care Costs. Health Serv Outcomes Res Methodol. 2000;1 (2):185-202.

30. Manning WG, Mullahy J. Estimating log models: to transform or not to transform? J Health Econ. 2001;20(4):461-94.

31. Diehr P, Yanez D, Ash A, Hornbrook M, Lin D. Methods for analyzing health care utilization and costs. Annu Rev Public Health. 1999;20(1):125-44.

32. Saultz JW, Lochner J. Interpersonal Continuity of Care and Care Outcomes: A Critical Review. Ann Fam Med. 2005;3(2):159-66.

33. Allard M, Jelovac I, Léger PT. Treatment and referral decisions under different physician payment mechanisms. J Health Econ. 2011;30(5):880-93.

34. Hunter DJ, Shortt SE, Walker PM, Godwin M. Family physician views about primary care reform in Ontario: a postal questionnaire. BMC Fam Pract. 2004;5(1):2.

35. Parker RM, Ratzan SC, Lurie N. Health Literacy: A Policy Challenge For Advancing High-Quality Health Care. Health Aff (Millwood). 2003;22(4):147-53.

36. Chalkley M, Malcomson JM. Contracting for health services when patient demand does not reflect quality. J Health Econ. 1998;17(1):1-19.

37. Choné P, Ma CA. Optimal Health Care Contract under Physician Agency. Ann Econ Stat. 2011;101/102:229-56.

38. Godager G, Wiesen D. 'Profit or patients' health benefit? Exploring the heterogeneity in physician altruism'. J Health Econ. 2013;32(6):1105-16.

39. Liu T, Ma CA. Health insurance, treatment plan, and delegation to altruistic physician. Financ Sect Perform Risk. 2013;85:79-96.

40. Laberge M, Wodchis WP, Barnsley J, Laporte A. Hospitalizations for ambulatory care sensitive conditions across primary care models in Ontario, Canada. Soc Sci Med. 2017;181:24-33.

\section{Submit your next manuscript to BioMed Central and we will help you at every step:}

- We accept pre-submission inquiries

- Our selector tool helps you to find the most relevant journal

- We provide round the clock customer support

- Convenient online submission

- Thorough peer review

- Inclusion in PubMed and all major indexing services

- Maximum visibility for your research

Submit your manuscript at www.biomedcentral.com/submit 